\title{
Neue Dächer nötig?
}

\section{Nach der Initiierung vieler kommunaler Lokale Agenda 21-Prozesse rückt die Raumebene Region derzeit stärker in den Blickpunkt. Implementierung und Ausge- staltung Nachhaltiger Regionalentwicklung innerhalb der Regionen gestalten sich vor Ort sehr heterogen. Ohne den vermessenen Anspruch einer starren Kategori- sierung versucht dieser Beitrag, die Konzepte zu systematisieren.}

$\mathrm{I}$ Von Reimar Molitor der aktuellen Diskussion um Nachhaltige Regionalentwicklung (NRE) ist eine gewisse Stagnationsphase in der anwendungsbezogenen Forschung eingetreten, die sich am besten an den unzähligen Kongressen und Workshops zum Austausch von Erfahrungen in NRE-Projekten ablesen läßt. Helge Majer bringt den derzeitigen Stand der Diskussion auf den Punkt, indem er feststellt, daß die Phase des ,Jagens und Sammelns von Erkenntnissen " über NRE vorbei sei (1). Doch was kommt danach? Gibt es trotz der Vielfalt der regionalen Ansätze zu Nachhaltiger Entwicklung Eckpunkte, die jenseits der Beschreibung von 'best practices' der Diskussion als ,Leitplanken“ dienen können? Es erscheint sinnvoll, sich den Projekten Nachhaltiger Regionalentwicklung von ihrem Ursprung her zu nähern. Diese auf den ersten Blick triviale Erkenntnis wird gerne vernachlässigt. Schnell wendet man sich Fragen der Resonanz und Evaluation von Projekten (oder derem bloßen ,Abfeiern") zu, ohne sich vorher mit den Ausgangsbedingungen und der Initiierungsebene beschäftigt zu haben, die wesentlich für die langfristige Etablierung des Prozesses NRE sind.

\section{Grundvoraussetzungen für die Etablierung Nachhaltiger Regionalentwicklung}

1. Verständigung iber den Ist-Zustand der Region - Etablierung einer 'corporate identity' für NRE

Dabei ist darauf zu achten, den Beitrag und Stand der jeweiligen Region bezüglich der ökologischen, ökonomischen, soziolen (Fehl-) Entwicklungen konsequent und sachlich zu benennen. Dieses Unterfangen gestaltet sich vor Ort sehr aufwendig und nicht selten sehr emotional. Es fehlt für viele Bereiche eine regionale Datenbasis, auf die man sich verständigen konn, Verursacher entziehen sich der Verantwortung für einen bestimmten regionolen Entwicklungszustand oder werten diesen anders, es kommen Ängste ob der Verschiebung von Macht und Einflußmöglichkeiten ouf etc. Aufgrund dieser Schwierigkeiten wird die Herstellung eines Konsens über den aktuellen Entwicklungszustand einer Region und dessen Abgleich mit den Anforderungen der Nachhaltigkeit gerne vernachlässigt

\section{Verständigung/Konsens ther den Soll-} Zustand der Region - Erarbeitung von regionalen Nachhaltigkeitspotentialen

Die Organisations- und Beteiligungsform der Konsensbildung für den (nochholigen) Soll-Zustand der Region hängt stark vom jeweiligen Typus der NRE ab. Es sollte prioritür darouf geachtet werden, regionale Nachhaltigkeitspotentiale so zu definieren, daß die regionalen Kräfte der Umsetzung diesen "Entwicklungskorridor"
(4) auch realistisch ausfïllen können. Notwendig ist hier die Unterscheidung zwischen kurzfristig angepeilten Zielen und Erfolgen, die für regionale Akteure nachahmenswert erscheinen $\left({ }_{n}\right.$ good proctices $\left.{ }^{\prime \prime}\right)$ und eine regionole Aufbruchstimmung erzeugen, sowie der langfristi: gen Strategie (regionales Entwicklungs-Leitbild).

\section{Verstetigung durch kurzfristige Erfolge und} langfristige Strategie - kontinuierliche Reflexion der Ziele

Da NRE als dynamischer Prozeß verstanden werden muß, der sich im Idealfall in bestimmten Zeitabstünden an wondelnde regionale Bedürnisse neu anpaft, kommt besonders der kontinuierlichen Reflexion des Prozesses eine Schlüsselrolle für die Etablierung zu. Dieses noch unzulänglich beachtete Merkmol wird in den laufenden Projekten ous unterschiedlichen Gründen vernachlässigt: die finanzielle Projektförderung läuft über einen zu kurzen Zeitraum, die Initiatoren scheuen eine unbequeme Infrogestellung „ihres" Projektes, das Planungs- und Entwicklungsdenken der Beteiligten ist eher linear ausgerichtet etc. Die Frage nach der kontinuierlichen Reflexion hängt auch eng mit der Frage der Bewertung bzw. Evaluation zusammen (vgl. den Beitrag von Fiebig in diesem Heft). Diesem Komplex des NREProzesses wird in Zukunft mehr Aufmerksamkeit gewidt. met werden, speziell unter Gesichtspunkten der Förderwürdigkeit von Projekten.

\section{Drei NRE-Typen}

Grundsätzlich kann man zwischen drei Typen von NRE unterscheiden (s. Abb. 1). Typ A beschreibt jene Ansätze, die einen „top-downapproach" haben. Ausgangspunkt für diesen Typ ist eine administrative Ebene, deren räumlicher Zuschnitt eine Region abbildet. Zu ihnen zählen Regionalverbände, Regionalkonferenzen, regionale Entwicklungsagenturen sowie Regionalforen. „Diese Formen der Kooperation zwischen Verbänden, Institutionen, Politik und Verwaltung beziehen sich in erster Linie auf die Erarbeitung von strukturpolitischen Leitbildern und die Abstimmung von Planungsprozessen, weniger auf die Erarbeitung und Durchfiuhrung konkreter Projekte“ (2). Die Motivation dieser ,intermediären Gruppen“ resultiert häufig aus der praktischen Absicht, in den jeweiligen Anforderungskatalog der EU-Förderkulisse zu passen oder politisch-motiviertes Regionenmarketing zu praktizieren. Große Gefahr dieses Typus ist die „Versandung“ des Prozesses im Stadium der Papierform. Der fuir eine Nachhaltige Entwicklung essentielle Faktor der Umsetzung wird oftmals nicht oder nur unzureichend erfiillt.

Typ B beschreibt jene Ansätze und Projekte, die aus dem weiten Bereich von Initiativen, Vereinen und anderen Formen von Zusammenschlüssen regionaler Akteure stammen. Ausgangspunkte dieses Typus sind überwiegend ökologische, ökonomische und/oder soziale „Schieflagen“ regionaler Entwicklung, die von Einzelakteuren oder einem Zusammenschluß von Akteuren aufgegriffen und thematisiert werden. Da in diesem Fall konkrete (sektorale) Bedürfnisse vor Ort als Initialzündung für NRE wirken, ist der Faktor Umsetzung dominantes Merkmal dieser Ansätze. In den meisten Fällen des Typus B wird die Raumebene Region erst im nachhinein flexibel bestimmt oder an eine bestehende regionale Raumstruktur angelehnt (vgl. den Beitrag von Nischwitz in diesem Heft). Die Thematik selbst bleibt jedoch im Mittelpunkt aller Aktivitäten, die Region bildet nur die räumliche Kulisse.

Der weitaus größte Anteil von Projekten und Ansätzen ist dem Typ C zuzuordnen, in dem sich Planungsinstitutionen mit regionalen Initiativen und Pionieren unter dem ,neuen Dach“ der NRE zusammenfinden. Diese Allianzen zwischen „top-down“ und ,bottom-up“Ansätzen bilden den gängigsten Typus der NRE, liegen doch genau hier die vielfach für eine NRE geforderten ,win-win Konstellationen“ (3). Auffallend hoch ist dabei der Zeitaufwand 


\section{Abb. 1: Typen Nachhaltiger Regionalentwicklung}

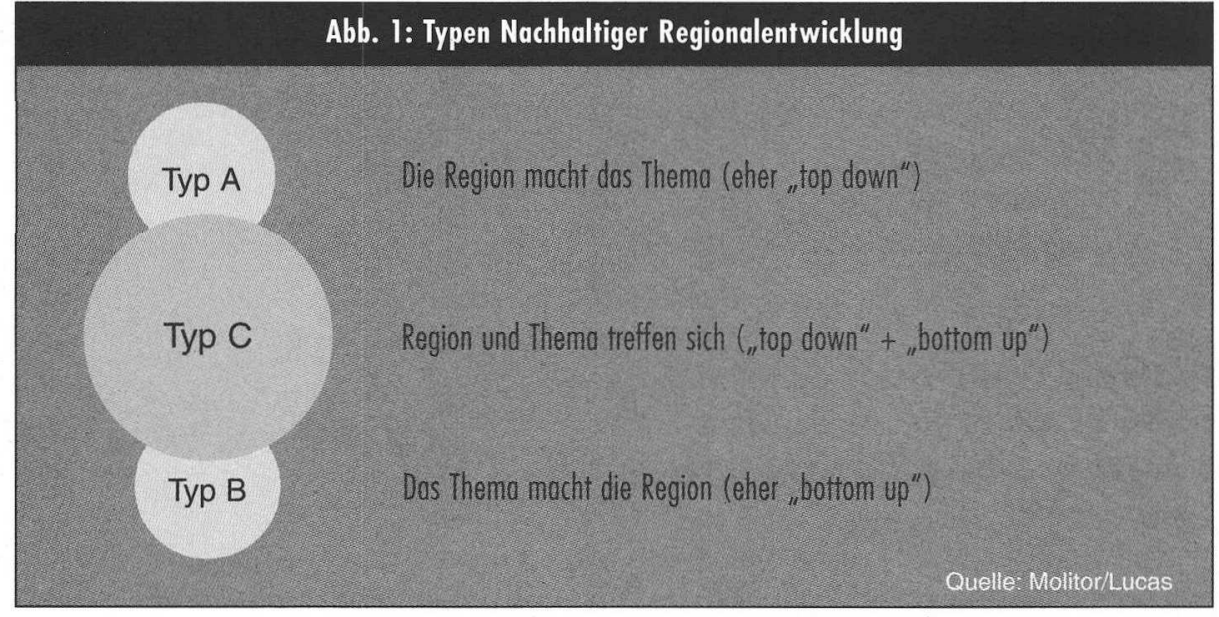

\begin{tabular}{|c|c|c|c|c|}
\hline 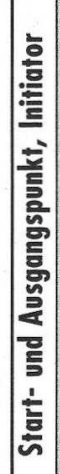 & $\begin{array}{l}\text { Programm } \\
\text { - integratives } \\
\text { Problemverständnis } \\
\text { - allgemeine Ziele } \\
\text { - raumbezogene Ziele } \\
\text { - Strukturierung von } \\
\text { Handlungsfeldern } \\
\text { - Akteursorientierung } \\
\text { - thematische Prioritöten } \\
\text {-... }\end{array}$ & $\begin{array}{l}\text { Prozeil } \\
\text { - Finanzierung } \\
\text { - Laufzeit (zeitiche Dimension) } \\
\text { - Träger } \\
\text { - Begleitung/Antrieb } \\
\text { - Kooperation } \\
\text { - Beteiligung } \\
\text { (Netzwerk, ...) } \\
\text { - Projekte } \\
\text {-... }\end{array}$ & $\begin{array}{l}\text { Überprüfung/ } \\
\text { Reflexion } \\
\text { Ziel-Mittel-Verhältnis } \\
\text { Ist-Zustand/Soll-Zustand } \\
\text { - ökologisch } \\
\text { - ökonomisch } \\
\text { - sozial } \\
\text { - räumlich } \\
\text { (Region) }\end{array}$ & $\begin{array}{l}\text { Wirkung } \\
\text { Evaluations- } \\
\text { projekte }\end{array}$ \\
\hline
\end{tabular}

Quelle: Molitor/Lucas

für die Initiierung, der mit dem „Beschnuppern" der verschiedenen am Prozeß teilnehmenden Gruppen und Individuen einhergeht. Dieses oftmals schwierige Unterfangen der Überwindung bestehender Gräben und Herstellung einer Vertrauensbasis innerhalb der Region ist ein wesentliches Element für die langfristige (und erfolgreiche) Verankerung von NRE.

\section{- Ebenen und Elemente von NRE}

Nachhaltige Regionalentwicklung unterliegt komplexen Bedingungen. Deswegen erscheint es sinnvoll, zwischen verschiedenen Ebenen und Elementen zu unterscheiden (s. Abb. 2). Die Ebene 'Start- und Ausgangspunkt, Initiator' ist mit der oben vorgenommenen Typisierung weitestgehend identisch. Hier bedingt die Motivation langfristig die Ausgestaltung von NRE. Die Ebene 'Programm' hat ihr Schlüsselelement in der thematischen Prioritätensetzung und der daran anschließenden Strukturierung von Handlungsfeldern. In vielen Projekten zeigt sich, daß die Überwindung der sektoralen Nischen ein Kernelement für die Etablierung von NRE ist. So verwundert es nicht, daß in der Praxis die Handlungsfelder in den meisten Fällen übersektoral strukturiert werden (,Neue Dächer“) (vgl. den Beitrag von Franck in diesem Heft). Die Ebene 'Prozeß' läßt sich nur schwer in ihre Einzelelemente auflösen, da deren Bezug untereinander zu stark ist. Eine wichtige Rolle spielt die Begleitung bzw. der stetige Antrieb von NRE. Rein projektbezogene Ansätze schaffen es oft nicht, den Prozeß über die Phase des Anfangsenthusiasmus hinaus innerhalb der Region langfristig „hoffähig“" zu machen bzw. zu verankern. Während die Ebenen 'Programm' und 'Prozeß' mit ihren dazugehörigen Elementen im wesentlichen durch den jeweiligen Typus der NRE vorgezeichnet sind, gestalten sich die Ebenen 'Überprïfung/Reflexion' und 'Wirkung' sehr diffus. So hängt die wichtige Bestimmung des Ziel-Mittel-Verhältnisses als Kernelement der Ebene 'Überprüfung/Reflexion' irgendwo zwischen der Suche nach quantitativen (harten) Indikatoren und der qualitativen Beschreibung des Lernprozesses der Akteure fest. Allerdings lassen sich einige Grundvoraussetzungen von NRE (vgl. Kasten) nennen.

\section{- Wirkung von NRE-Prozessen}

Die Bandbreite dessen, was NRE eigentlich bewirkt bzw. bewirken soll, ist so vielfältig wie die unterschiedlichen Interessenlagen innerhalb der Regionen selbst. Dementsprechend schwierig gestaltet sich auch die Evaluation von NRE und deren Erfolgsbedingungen. Der Forderung des Umweltbundesamtes, Nachhaltigkeit meßbar zu machen (5), kann für den Prozeß NRE allenfalls mit Instrumenten der qualitativen Sozialforschung nachgekommen werden, da sich die Wirkung langfristig nur aus den Lernprozessen der regionalen Akteure heraus entfalten und verstetigen wird.

NRE sollte deshalb im Idealfall als anspruchsvoller, demokratischer Prozeß der Konsensbildung über regionale Entwicklungszustände verstanden werden, der zeitlich und räumlich betrachtet dynamisch ist und dabei den Anforderungen der Nachhaltigkeit entspricht.

\section{Anmerkungen}

(1) Majer, Helge; Kai, Weinmüller: Umsetzung von regionalem Wirtschaften. Ergebnisbericht des Expertenworkshops „Regionale Ansätze nachhaltigen Wirtschaftens" des Bundesministeriums für Bildung, Wissenschaft, Forschung und Technologie. Stuttgart 1997. Vorwort.

(2) Kleine-Limberg, Wolfgang: Chancen und Grenzen regionaler Selbstorganisation. In: R. Danielzyk, C. Deppe,

I. Mose (Hrsg.): Von der eigenständigen zur nachhaltigen Regionalentwicklung. Bremen 1998, S. 25.

(3) Majer, Weinmüller, a.a.0.

(4) Vgl. zum Begriff des "Entwicklungskorridors" Klemmer, Paul: Wege aus der Krise? In: reporter spezialheft 2.

Frankfurt a.M. 1995, S. 20.

(5) Umweltbundesamt (Hrsg.): Nachhaltiges Deutschland. Wege zu einer dauerhaft umweltgerechten Entwicklung. Berlin 1997, S. 16.

Der Autor
Reimar Molitor ist freier Mitarbeiter am IÖW,
Regionalbüro Nordrhein-Wesifalen. Er promoviert
derzeit in Irland zum Thema 'Nachhaltige Regional.
entwicklung in Europa'.
Kontakt: Ardglassan, Crossakiel, Kells,
County Meath, Republic of Ireland,
Tel. 00353/ (0) $46 / 43808$,
E-mail: doranb@indigo.ie oder
IÖW, Völklinger Sir.9, 42285 Wuppertal

\begin{tabular}{|c|}
\hline Diskussionsforum \\
Die Debatte über Nachhaltige Regional- \\
entwicklung soll fortgeführt werden. Das \\
IÖW und die Redaktion freuen sich \\
daher über kurze Beiträge (max. 1 Heft- \\
seite, das entspricht 5000 Zeichen), die \\
auf die Artikel dieses Schwerpunktes \\
reagieren.
\end{tabular}


(c) 20I0 Authors; licensee IÖW and oekom verlag. This is an article distributed under the terms of the Creative Commons Attribution Non-Commercial No Derivates License (http://creativecommons.org/licenses/by-nc-nd/3.o/), which permits unrestricted use, distribution, and reproduction in any medium, provided the original work is properly cited. 